\title{
Urban stream Osówka in Szczecin - how efficiently as possible should the variability of the stream water quality be studied along the road of runoff in time?
}

\author{
TyMOTEUsz MilleR, GORZYSŁAW POLESZCZUK
}

Department of Chemistry and Natural Waters Management, Institute for Research on Biodiversity, Faculty of Biology, Szczecin University, ul. Z. Felczaka 3C, 71-412 Szczecin, Poland, e-mail: polesz@univ.szczecin.pl.

Keywords Urban stream, Osówka Stream, chemometric analysis, linear regression, factor analysis, cluster analysis, water quality

Abstract The water quality of the stream Osówka in the NW part of the Szczecin city was studied. Water samples were collected in four seasons of 2014 - January, April, July and October in 6 sampling stations, from station near the source of the stream - to inflow of Osówka stream water to the lake Rusalka. 20 selected water chemistry indices were measured. The aim of discernment variability of water quality along stream runoff path during studied period chemometric procedures were conducted: cluster analysis (CA), calculations of linear regression equation $y_{i}=\bar{y}_{i}+\sum_{j=1}^{6} \alpha_{j} S_{j}+\sum_{k=1}^{4} \beta_{k} T_{k}+S E E$ for selected water quality indices $\left(\mathrm{y}_{\mathrm{i}}\right)$, where $\mathrm{S}_{\mathrm{j}}$ and $\mathrm{T}_{\mathrm{k}}$ - are sampling stations and sampling terms numbers - respectively, and factor analysis (FA). It has been shown significant variability of Osówka water quality along the runoff path and established what parameters characterizing the variability of stream water chemistry should be examined in the places of indicated sampling stations - the same as in the studies the quantities of terms of sampling in 4 consecutive seasons.

\section{Strumień Osówka w Szczecin - jak najefektywniej badać regularnie w czasie zmienność jakości wód tego strumienia wzdłuż drogi ich spływu?}

Słowa kluczowe ciek wodny w mieście, ciek Osówka, analiza chemometryczna, regresja liniowa, analiza czynnikowa, analiza skupień, jakość wody

Streszczenie Badano jakość wód strumienia Osówka w NW części miasta Szczecina. Próbki wody do badań pobierano w czterech terminach - styczeń, kwiecień, lipiec i październik 2014 roku w miejscu 6 stacji, poczynając od ujęcia w pobliżu źródła strumienia - aż do dopływu wód Osówki do jeziora Rusałka. Oznaczano 20 wybranych wskaźników jakości wód. Celem rozeznania zmienności jakości wód wzdłuż drogi spływu w okresie badawczym przeprowadzono studia chemometryczne posługując się procedurami: CA, obliczano równania regresji $y_{i}=\overline{y_{i}}+\sum_{j=1}^{6} \alpha_{j} S_{j}+\sum_{k=1}^{4} \beta_{k} T_{k}+S E E$ dla poszczególnych wskaźników jakości wód $\left(\mathrm{y}_{\mathrm{i}}\right)$, gdzie $\mathrm{S}_{\mathrm{j}} \mathrm{i} \mathrm{T}_{\mathrm{k}}$ - to numery stacji i terminów poboru próbek - odpowiednio, a także FA. Wykazano znaczną zmienność jakości wód Osówki wzdłuż drogi spływu i ustalono, jakie parametry charakteryzujące zmienność chemizmu wód strumienia powinny być badane w miejscach 
wskazanych stacji pomiarowych - przy identycznej jak w przeprowadzonych badaniach ilościach terminów poboru próbek w następujących po sobie 4 porach roku.

\section{Introduction}

Examination of streams and rivers water flowing through urban areas, which is associated with very significant changes in the quality of waters - contaminated with sewage during flows through city areas, becoming an increasingly important issue in relation to their progressive degradation (Liu et al. 2014; Wilkins et al. 2015; Huang et al. 2016). In many papers this problem is known as „urban stream syndrome” (Liu et al. 2014; Wilkins et al. 2015; Huang et al. 2016).

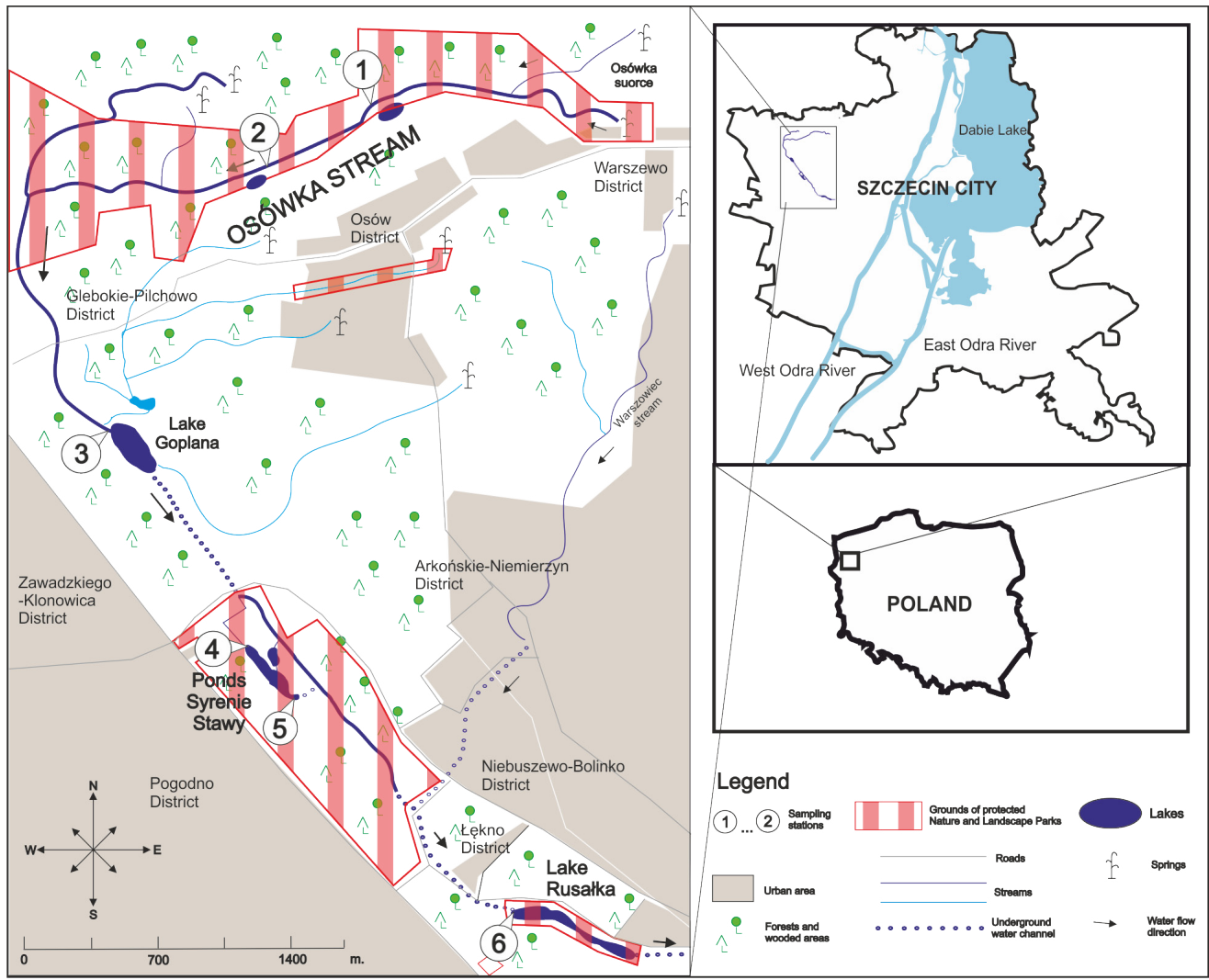

Figure 1. Osówka stream in Szczecin city (NW-Poland)

Osówka stream located in the north-west part the Szczecin city (Fig. 1) is a stream around which are located areas of nature conservation and recreation. Two nature and landscape protection complexes were established here: "Dolina Siedmiu Młynów i źródło strumienia Osówka" and "Zespół Parków Kasprowicza-Arkoński" (Uchwała 1994, Stan 2004). At the same time stream Osówka is included in the municipal rain sewer system and receives rainwater and sewage, 
unfortunately, which gradually run down with natural watercourse and by open concrete channels or underground concrete channels to Western Odra. Studies having the character of random studies of Osówka stream waters and inflow water to Osówka have been published - so far in a relatively small number of publications, eg. Raczńska (2012a \& b), Miller et al. (2014 a \& b).

\section{Characteristics of Osówka stream}

The total length of the Osówka stream in the north-west part of Szczecin is about $13 \mathrm{~km}$. Sources of the stream are located in the Warszewkie hills on the southern slope of Lisia Mountain (108 $\mathrm{m}$ asl). From the source - through the Valley of the Seven Mills waters of Osówka flow down as a rapid stream to Lake Goplana $(5.15 \mathrm{~km})$. Above Goplana lake to Osówka inflow waters of two relatively small streams: Bystry Potok $(0.75 \mathrm{~km})$ and Jasmudzka Struga $(3.73 \mathrm{~km})$. Near the Osówka water outflow from the Goplana lake - is an underground inflow the Arkonka stream. Osówka water flows out of the Lake Goplana $(5.52 \mathrm{~km})$ with an underground channel with a length of approx. $700 \mathrm{~m}$. After that stream bed separates into two - already on the surface - above the Sytrenie Stawy Ponds $(6.72 \mathrm{~km})$. From the Ponds Syrenie Stawy the Osówka waters outflows with an open channel and when combined with the "relief" channel (bypassing Syrenie Stawy) flow down further with underground channel to the Rusałka lake. Along the way, $(7.60 \mathrm{~km})$ into an underground channel, falls water of the Warszowiec stream. At $8.63 \mathrm{~km}$ underground channel ends in the Rusalka lake. Osówka stream water after passing the Rusalka lake outflows from the reservoir $(\mathrm{km} \mathrm{9.34)}$ and falls further with underground channel to Western Odra (Report 2004).

\section{Material}

Water samples of Osówka stream were collected at six sampling stations located respectively at $1.57 ; 2.27 ; 5.52 ; 6.72 ; 7.00$ and $8.64 \mathrm{~km}$ from the source - recognized as the beginning of the stream. Water samples were collected from the stream according to the rules specified in PNISO 5667-6: 2003, and from the water reservoirs through which waters of Osówka flowed - using a model Bucket - from the surface waters from a depth of about $25 \mathrm{~cm}$ below the water-according to PN-ISO 5667-4: 2003. Water sampling was conducted during the four seasons, ie. during the period of winter - on 15.01.2014, the period of spring - 16.04.2014, summer - 15.07.2014, and autumn - 15.10.2014. Examination of urban streams in different seasons is recommended in a number of publications (Sun et al. 2016; Ledford, Lautz 2016; Pratt, Chang 2012).

In studies of water streams and rivers flowing through the cities chemometric procedures are a fundamental tool, eg. Muangthong, Shrestha (2015), Haque et al. (2016), Hamid et al. (2016). In our work measurement data were analyzed using cluster analysis (CA), linear regression equation was calculated: $y_{i}=\bar{y}_{i}+\sum_{j=1}^{6} \alpha_{j} S_{j}+\sum_{k=1}^{4} \beta_{k} T_{k}+S E E$ showing the relationship of the following investigated water quality indices from the average values of all measurements $\overline{y_{i}}$ from the sampling station and terms of samples collection - where in equation the next values of $S_{j}$ take a value 1 for $\mathrm{j}$-th sampling station and value 0 for next station numbers, similarly $-\mathrm{T}_{\mathrm{k}}$ take a value 1 for $\mathrm{k}$-th term and 0 - for next terms. Regression equations were calculated using the method of stepwise deletion and addition a priori (Forward) (Stanisz 2007). Subsequent equations were calculated using the sequence Fisher-Snedecor test using as a criterion for rejection of the variables test values $-\mathrm{F}=4$. Last chemometric method conducted was factor analysis (FA) - using PCA as an 
extraction method, and after PCA - normalized varimax rotation - for all the data collected during the research. Before factor analysis was conducted Kaiser-Meyer-Olkin (KMO) and Bartlett's sphericity tests were performer to confirm adequacy of the collected data to FA. These procedures made it possible to determine which parameters have changed with the statistically significant way and identify which indices should be studied regularly in order to comprehensively examine changes in water quality over time along the path of runoff. All calculations were performed using the Statistica 12.0 PL and Statrgaphics Centurion XVII.

Analysis of selected physical and chemical indices of water quality of Osówka stream were performed with the methods recommended by the APHA (2012) according to the analytical procedures contained in the Polish Standards (Polskie Normy). At the sampling stations the temperature of water by mercury thermometers and $\mathrm{pH}$ - potentiometrically were determined, as well as total alkalinity (Alk), converted to the concentration of $\mathrm{HCO}_{3}{ }^{-}$- by acidimetry titration. Separate water samples were taken for assessment of the concentration of dissolved oxygen (DO) and 5 day biochemical oxygen demand $\left(\mathrm{BOD}_{5}\right)$. Designation of dissolved oxygen in water was performed in the laboratory by Winkler method. Collected Additional samples of water for analysis other selected water quality indices were collected to determine: without filtration - COD-Cr, COD-Mn and total concentration of nitrogen (TN) and phosphorus (TN) and also total concentrations of iron $\left(\mathrm{Fe}_{\text {tot }}\right)$ and manganese $\left(\mathrm{Mn}_{\text {tot }}\right)$; with filtration through filters having a diameter of micropores $0,45^{\cdot} \cdot 10^{-9} \mathrm{~m}-\mathrm{NO}_{3}^{-}, \mathrm{NO}_{2}^{-}$and $\mathrm{NH}_{4}^{+}, \mathrm{PO}_{4}^{3-}(\mathrm{SRP}), \mathrm{Ca}^{2+}, \mathrm{Mg}^{2+}, \mathrm{Cl}^{-}, \mathrm{SO}_{4}^{2-}$.

All analyzes performed in the laboratory within 24 hours after sampling. Waster saturation by $\mathrm{O}_{2}$ (WS) was calculated according to Nemerow (1985). COD-Cr i COD-Mn - were performed by titration method. Concentrations of $\mathrm{NO}_{3}^{-}, \mathrm{NO}_{2}^{-}, \mathrm{NH}_{4}^{+}$and SRP (soluble reactive orthophosphates (V)) - by colorimetric methods as well as total concetrations of TN and TP - after samples mineralization. By titration method with EDTA - total concentration of calcium and magnesium was measured. Concentration of chlorides by argentometric Mohr method. Gravimetric method was used to define concentration of sulfates (VI). Total concentrations of iron and manganese were indicated by colorimetric method. Similar water quality indices in urban streams were examined, eg. at work Chang (2008).

\section{Results and discussion}

The test results of water samples taken in sampling stations $\mathrm{S}_{1}-\mathrm{S}_{6}$ located respectively at km1.43; 2.27; 5.52; 6.72; 7.00 and 8.64 of the stream are shown in Tab. 1-4. Fig. 2 and 3.

Table 1. The values of selected water quality indices for the subsequent water sampling stations $\left(S_{j}\right)$ on the Osówka stream in January of 2014. Sampling date: 15.01.2014

\begin{tabular}{|l|l|l|c|c|c|c|c|c|}
\hline \multirow{2}{*}{ L.p. } & \multirow{2}{*}{$\begin{array}{c}\text { Water quality } \\
\text { indices }\end{array}$} & \multicolumn{2}{|c|}{ Units } & \multicolumn{7}{|c|}{ Stacja $\left(S_{j}\right)$} \\
\cline { 4 - 10 } & & \multicolumn{1}{|c|}{3} & $\mathrm{~S}_{1}$ & $\mathrm{~S}_{2}$ & $\mathrm{~S}_{3}$ & $\mathrm{~S}_{4}$ & $\mathrm{~S}_{5}$ & $\mathrm{~S}_{6}$ \\
\hline 1 & \multicolumn{1}{c}{3} & 4 & 5 & 6 & 7 & 8 & 9 \\
\hline 1 & Temperature & ${ }^{\circ} \mathrm{C}$ & 4.3 & 1.5 & 0.6 & 0.6 & 0.4 & 5.3 \\
\hline 2 & $\mathrm{pH}$ & $\mathrm{pH} \mathrm{units}$ & 7.80 & 8.29 & 7.66 & 7.20 & 7.26 & 8.70 \\
\hline 3 & COD-Mn & $\mathrm{mg} \mathrm{O}_{2} \cdot \mathrm{dm}^{-3}$ & 7.4 & 7.7 & 8.9 & 9.5 & 7.2 & 8.8 \\
\hline 4 & COD-Cr & $\mathrm{mg} \mathrm{O}_{2} \cdot \mathrm{dm}^{-3}$ & 13.4 & 20.2 & 87.6 & 215.0 & 67.4 & 303.0 \\
\hline
\end{tabular}




\begin{tabular}{|c|c|c|c|c|c|c|c|c|}
\hline 1 & 2 & 3 & 4 & 5 & 6 & 7 & 8 & 9 \\
\hline 5 & $\mathrm{BOD}_{5}$ & $\mathrm{mg} \mathrm{O}_{2} \cdot \mathrm{dm}^{-3}$ & 2.6 & 2.0 & 8.0 & 2.0 & 2.6 & 4.0 \\
\hline 6 & $\mathrm{DO}$ & $\mathrm{mg} \mathrm{O}_{2} \cdot \mathrm{dm}^{-3}$ & 8.6 & 10.8 & 10.4 & 2.0 & 2.6 & 5.0 \\
\hline 7 & WS & $\%$ & 66.0 & 86.4 & 83.1 & 16.0 & 20.8 & 39.1 \\
\hline 8 & $\mathrm{NO}_{3}^{-}$ & $\mathrm{mg} \mathrm{NO}_{3}^{-} \cdot \mathrm{dm}^{-3}$ & 0.30 & 0.24 & 0.42 & 0.77 & 1.00 & 0.99 \\
\hline 9 & $\mathrm{NO}_{2}^{-}$ & $\mathrm{mg} \mathrm{NO}_{2}^{-} \cdot \mathrm{dm}^{-3}$ & 0.088 & 0.013 & 0.005 & 0.314 & 0.127 & 0.030 \\
\hline 10 & $\mathrm{NH}_{4}^{+}$ & $\mathrm{mg} \mathrm{NH}_{4}^{+} \cdot \mathrm{dm}^{-3}$ & 0.31 & 0.21 & 0.28 & 0.32 & 0.39 & 0.34 \\
\hline 11 & $\mathrm{TN}$ & $\mathrm{mg} \mathrm{N} \cdot \mathrm{dm}^{-3}$ & 0.65 & 0.52 & 0.83 & 1.51 & 1.52 & 1.91 \\
\hline 12 & SRP & 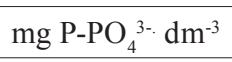 & 0.07 & 0.02 & 0.04 & 0.35 & 0.15 & 0.18 \\
\hline 13 & $\mathrm{TP}$ & $\mathrm{mg} \mathrm{P}-\mathrm{PO}_{4}^{3--} \mathrm{dm}^{-3}$ & 0.13 & 0.07 & 0.10 & 0.44 & 0.92 & 0.41 \\
\hline 14 & $\mathrm{Ca}^{2+}$ & $\mathrm{mg} \mathrm{Ca} \cdot \mathrm{dm}^{-3}$ & 74 & 58 & 51 & 67 & 105 & 136 \\
\hline 15 & $\mathrm{Mg}^{2+}$ & $\mathrm{mg} \mathrm{Mg} \cdot \mathrm{dm}^{-3}$ & 5 & 6 & $<5$ & 6 & 19 & 16 \\
\hline 16 & $\mathrm{Cl}^{-}$ & $\mathrm{mg} \mathrm{Cl} \cdot \mathrm{dm}^{-3}$ & 27 & 22 & 21 & 34 & 31 & 32 \\
\hline 17 & $\mathrm{SO}_{4}^{2-}$ & $\mathrm{mg} \mathrm{SO}_{4} \cdot \mathrm{dm}^{-3}$ & 13 & 10 & 14 & 81 & 77 & 69 \\
\hline 18 & $\mathrm{HCO}_{3}^{-}$ & $\mathrm{mg} \mathrm{HCO} \cdot \mathrm{dm}^{-3}$ & 225 & 225 & 175 & 150 & 225 & 275 \\
\hline 19 & $\mathrm{Fe}_{\text {tot }}$ & $\mathrm{mg} \mathrm{Fe} \cdot \mathrm{dm}^{-3}$ & 0.09 & 0.08 & 0.01 & 1.33 & 1.33 & 0.16 \\
\hline 20 & $\mathrm{Mn}_{\text {tot }}$ & $\mathrm{mg} \mathrm{Mn} \cdot \mathrm{dm}^{-3}$ & 0.01 & 0.10 & 0.01 & 0.01 & 0.01 & 0.01 \\
\hline
\end{tabular}

Table 2. The values of selected water quality indices for the subsequent water sampling stations $\left(S_{j}\right)$ on the Osówka stream in April of 2014. Sampling date: 16.04.2014

\begin{tabular}{|c|c|c|c|c|c|c|c|c|}
\hline \multirow{2}{*}{ L.p. } & \multirow{2}{*}{$\begin{array}{l}\text { Water quality } \\
\text { indices }\end{array}$} & \multirow{2}{*}{ Units } & \multicolumn{6}{|c|}{$\operatorname{Stacja}\left(S_{j}\right)$} \\
\hline & & & $\mathrm{S}_{1}$ & $\mathrm{~S}_{2}$ & $\mathrm{~S}_{3}$ & $\mathrm{~S}_{4}$ & $\mathrm{~S}_{5}$ & $\mathrm{~S}_{6}$ \\
\hline 1 & 2 & 3 & 4 & 5 & 6 & 7 & 8 & 9 \\
\hline 1 & Temperature & ${ }^{\circ} \mathrm{C}$ & 4.8 & 5.0 & 4.6 & 5.1 & 5.2 & 3.8 \\
\hline 2 & $\mathrm{pH}$ & $\mathrm{pH}$ units & 8.16 & 8.67 & 8.02 & 7.54 & 8.05 & 7.95 \\
\hline 3 & COD-Mn & $\mathrm{mg} \mathrm{O}_{2} \cdot \mathrm{dm}^{-3}$ & 9.4 & 10.0 & 9.1 & 10.1 & 10.0 & 11.3 \\
\hline 4 & $\mathrm{COD}-\mathrm{Cr}$ & $\mathrm{mg} \mathrm{O}_{2} \cdot \mathrm{dm}^{-3}$ & 66.3 & 70.2 & 63.9 & 213.0 & 119.0 & 142.0 \\
\hline 5 & $\mathrm{BOD}_{5}$ & $\mathrm{mg} \mathrm{O}_{2} \cdot \mathrm{dm}^{-3}$ & 2.2 & 2.3 & 5.4 & 3.5 & 3.2 & 3.8 \\
\hline 6 & DO & $\mathrm{mg} \mathrm{O}_{2} \cdot \mathrm{dm}^{-3}$ & 12.4 & 13.5 & 12.8 & 3.5 & 4.2 & 8.0 \\
\hline 7 & WS & $\%$ & 99.1 & 108.0 & 102.3 & 28.0 & 33.6 & 60.6 \\
\hline 8 & $\mathrm{NO}_{3}^{-}$ & $\mathrm{mg} \mathrm{NO}_{3}^{-\cdot} \mathrm{dm}^{-3}$ & 0.40 & 0.42 & 0.38 & 0.64 & 0.81 & 0.72 \\
\hline 9 & $\mathrm{NO}_{2}^{-}$ & $\mathrm{mg} \mathrm{NO}_{2} \cdot \mathrm{dm}^{-3}$ & 0.144 & 0.152 & 0.139 & 0.231 & 0.118 & 0.025 \\
\hline 10 & $\mathrm{NH}_{4}^{+}$ & $\mathrm{mg} \mathrm{NH}_{4}^{++} \mathrm{dm}^{-3}$ & 0.51 & 0.54 & 0.49 & 0.82 & 1.02 & 0.85 \\
\hline 11 & $\mathrm{TN}$ & $\mathrm{mg} \mathrm{N} \cdot \mathrm{dm}^{-3}$ & 2.06 & 2.18 & 1.99 & 3.32 & 3.11 & 2.60 \\
\hline 12 & SRP & $\mathrm{mg} \mathrm{P}^{-\mathrm{PO}_{4}}{ }_{4}^{3-} \mathrm{dm}^{-3}$ & 0.17 & 0.18 & 0.17 & 0.28 & 0.21 & 0.20 \\
\hline 13 & $\mathrm{TP}$ & $\mathrm{mg} \mathrm{P}-\mathrm{PO}_{4}^{3-\cdot} \mathrm{dm}^{-3}$ & 0.64 & 0.68 & 0.62 & 1.04 & 1.15 & 0.65 \\
\hline 14 & $\mathrm{Ca}^{2+}$ & $\mathrm{mg} \mathrm{Ca} \cdot \mathrm{dm}^{-3}$ & 57 & 60 & 55 & 91 & 103 & 123 \\
\hline
\end{tabular}




\begin{tabular}{|c|c|c|c|c|c|c|c|c|}
\hline 1 & 2 & 3 & 4 & 5 & 6 & 7 & 8 & 9 \\
\hline 15 & $\mathrm{Mg}^{2+}$ & $\mathrm{mg} \mathrm{Mg} \cdot \mathrm{dm}^{-3}$ & $<5$ & $<5$ & $<5$ & 5 & 10 & 8 \\
\hline 16 & $\mathrm{Cl}^{-}$ & $\mathrm{mg} \mathrm{Cl} \cdot \mathrm{dm}^{-3}$ & 19 & 20 & 19 & 31 & 27 & 32 \\
\hline 17 & $\mathrm{SO}_{4}^{2-}$ & $\mathrm{mg} \mathrm{SO}_{4} \cdot \mathrm{dm}^{-3}$ & 33 & 35 & 32 & 53 & 49 & 41 \\
\hline 18 & $\mathrm{HCO}_{3}^{-}$ & $\mathrm{mg} \mathrm{HCO} \cdot \mathrm{dm}^{-3}$ & 200 & 225 & 175 & 200 & 225 & 250 \\
\hline 19 & $\mathrm{Fe}_{\text {tot }}$ & $\mathrm{mg} \mathrm{Fe} \cdot \mathrm{dm}^{-3}$ & 0.43 & 0.45 & 0.41 & 0.69 & 0.60 & 0.40 \\
\hline 20 & $\mathrm{Mn}_{\text {tot }}$ & $\mathrm{mg} \mathrm{Mn} \cdot \mathrm{dm}^{-3}$ & 0.09 & 0.09 & 0.08 & 0.14 & 0.07 & 0.03 \\
\hline
\end{tabular}

Table 3. The values of selected water quality indices for the subsequent water sampling stations $\left(S_{j}\right)$ on the Osówka stream in July of 2014. Sampling date: 15.07.2014

\begin{tabular}{|c|c|c|c|c|c|c|c|c|}
\hline \multirow{2}{*}{ L.p. } & \multirow{2}{*}{$\begin{array}{l}\text { Water quality } \\
\text { indices }\end{array}$} & \multirow{2}{*}{ Units } & \multicolumn{6}{|c|}{ Stacja $\left(S_{j}\right)$} \\
\hline & & & $\mathrm{S}_{1}$ & $\mathrm{~S}_{2}$ & $\mathrm{~S}_{3}$ & $\mathrm{~S}_{4}$ & $\mathrm{~S}_{5}$ & $\mathrm{~S}_{6}$ \\
\hline 1 & Temperature & ${ }^{\circ} \mathrm{C}$ & 12.5 & 13.2 & 12.1 & 13.4 & 13.5 & 12.1 \\
\hline 2 & $\mathrm{pH}$ & $\mathrm{pH}$ units & 8.03 & 8.53 & 7.89 & 7.89 & 8.40 & 8.30 \\
\hline 3 & COD-Mn & $\mathrm{mg} \mathrm{O}_{2} \cdot \mathrm{dm}^{-3}$ & 8.8 & 9.3 & 8.5 & 9.4 & 9.3 & 10.6 \\
\hline 4 & $\mathrm{COD}-\mathrm{Cr}$ & $\mathrm{mg} \mathrm{O}_{2} \cdot \mathrm{dm}^{-3}$ & 56.4 & 59.7 & 54.3 & 181.0 & 87.0 & 110.0 \\
\hline 5 & $\mathrm{BOD}_{5}$ & $\mathrm{mg} \mathrm{O}_{2} \cdot \mathrm{dm}^{-3}$ & 0.3 & 0.3 & 7.7 & 0.5 & 0.5 & 4.3 \\
\hline 6 & DO & $\mathrm{mg} \mathrm{O}_{2} \cdot \mathrm{dm}^{-3}$ & 12.3 & 8.6 & 8.0 & 0.5 & 0.5 & 4.3 \\
\hline 7 & WS & $\%$ & 98.3 & 68.4 & 63.9 & 4.0 & 4.0 & 39.7 \\
\hline 8 & $\mathrm{NO}_{3}^{-}$ & $\mathrm{mg} \mathrm{NO}_{3}^{-} \cdot \mathrm{dm}^{-3}$ & 0.52 & 0.55 & 0.50 & 0.84 & 1.01 & 0.92 \\
\hline 9 & $\mathrm{NO}_{2}^{-}$ & $\mathrm{mg} \mathrm{NO}_{2}^{-} \cdot \mathrm{dm}^{-3}$ & 0.147 & 0.156 & 0.068 & 0.236 & 0.123 & 0.030 \\
\hline 10 & $\mathrm{NH}_{4}^{+}$ & $\mathrm{mg} \mathrm{NH}_{4}^{+} \cdot \mathrm{dm}^{-3}$ & 0.50 & 0.53 & 0.48 & 0.80 & 1.00 & 0.83 \\
\hline 11 & $\mathrm{TN}$ & $\mathrm{mg} \mathrm{N} \cdot \mathrm{dm}^{-3}$ & 2.18 & 2.31 & 2.10 & 3.50 & 3.29 & 2.78 \\
\hline 12 & SRP & mg P-PO ${ }_{4}^{3-} \mathrm{dm}^{-3}$ & 0.31 & 0.39 & 0.30 & 0.50 & 0.57 & 0.56 \\
\hline 13 & $\mathrm{TP}$ & $\mathrm{mg} \mathrm{P}^{-} \mathrm{PO}_{4}{ }^{3--} \mathrm{dm}^{-3}$ & 1.15 & 1.22 & 1.11 & 1.85 & 2.06 & 1.56 \\
\hline 14 & $\mathrm{Ca}^{2+}$ & $\mathrm{mg} \mathrm{Ca} \cdot \mathrm{dm}^{-3}$ & 49 & 51 & 47 & 78 & 90 & 110 \\
\hline 15 & $\mathrm{Mg}^{2+}$ & $\mathrm{mg} \mathrm{Mg} \cdot \mathrm{dm}^{-3}$ & $<5$ & $<5$ & $<5$ & $<5$ & 7 & 5 \\
\hline 16 & $\mathrm{Cl}^{-}$ & $\mathrm{mg} \mathrm{Cl} \cdot \mathrm{dm}^{-3}$ & 18 & 19 & 17 & 29 & 25 & 30 \\
\hline 17 & $\mathrm{SO}_{4}^{2-}$ & $\mathrm{mg} \mathrm{SO}_{4} \cdot \mathrm{dm}^{-3}$ & 31 & 33 & 30 & 50 & 46 & 38 \\
\hline 18 & $\mathrm{HCO}_{3}^{-}$ & $\mathrm{mg} \mathrm{HCO} \cdot \mathrm{dm}^{-3}$ & 200 & 225 & 150 & 175 & 225 & 250 \\
\hline 19 & $\mathrm{Fe}_{\text {tot }}$ & $\mathrm{mg} \mathrm{Fe} \cdot \mathrm{dm}^{-3}$ & 0.34 & 0.36 & 0.33 & 0.55 & 0.46 & 0.26 \\
\hline 20 & $\mathrm{Mn}_{\text {tot }}$ & $\mathrm{mg} \mathrm{Mn} \cdot \mathrm{dm}^{-3}$ & 0.07 & 0.08 & 0.07 & 0.12 & 0.05 & 0.01 \\
\hline
\end{tabular}


Table 4. The values of selected water quality indices for the subsequent water sampling stations $\left(S_{j}\right)$ on the Osówka stream in October of 2014. Sampling date: 15.10.2014

\begin{tabular}{|c|c|c|c|c|c|c|c|c|}
\hline \multirow{2}{*}{ L.p. } & \multirow{2}{*}{$\begin{array}{l}\text { Water } \\
\text { quality } \\
\text { indices }\end{array}$} & \multirow{2}{*}{ Units } & \multicolumn{6}{|c|}{ Stacja $\left(S_{j}\right)$} \\
\hline & & & $\mathrm{S}_{1}$ & $\mathrm{~S}_{2}$ & $\mathrm{~S}_{3}$ & $\mathrm{~S}_{4}$ & $\mathrm{~S}_{5}$ & $\mathrm{~S}_{6}$ \\
\hline 1 & Temperature & ${ }^{\circ} \mathrm{C}$ & 13.7 & 14.5 & 13.2 & 14.7 & 14.8 & 13.4 \\
\hline 2 & $\mathrm{pH}$ & $\mathrm{pH}$ units & 7.65 & 8.03 & 7.43 & 7.62 & 8.13 & 8.03 \\
\hline 3 & COD-Mn & $\mathrm{mg} \mathrm{O}_{2} \cdot \mathrm{dm}^{-3}$ & 9.4 & 10.0 & 9.1 & 10.1 & 10.0 & 11.3 \\
\hline 4 & COD-Cr & $\mathrm{mg} \mathrm{O}_{2} \cdot \mathrm{dm}^{-3}$ & 60.7 & 64.3 & 58.5 & 195.0 & 101.0 & 124.0 \\
\hline 5 & $\mathrm{BOD}_{5}$ & $\mathrm{mg} \mathrm{O}_{2} \cdot \mathrm{dm}^{-3}$ & 0.7 & 0.8 & 4.5 & 1.2 & 1.9 & 5.1 \\
\hline 6 & $\mathrm{DO}$ & $\mathrm{mg} \mathrm{O}_{2} \cdot \mathrm{dm}^{-3}$ & 14.3 & 8.4 & 7.8 & 1.2 & 1.9 & 5.7 \\
\hline 7 & WS & $\%$ & 114.3 & 67.1 & 62.4 & 9.6 & 15.2 & 54.4 \\
\hline 8 & $\mathrm{NO}_{3}^{-}$ & $\mathrm{mg} \mathrm{NO}_{3}^{-} \cdot \mathrm{dm}^{-3}$ & 0.17 & 0.18 & 0.42 & 1.27 & 0.44 & 0.35 \\
\hline 9 & $\mathrm{NO}_{2}^{-}$ & $\mathrm{mg} \mathrm{NO}_{2}^{-} \cdot \mathrm{dm}^{-3}$ & 0.164 & 0.174 & 0.158 & 0.264 & 0.151 & 0.058 \\
\hline 10 & $\mathrm{NH}_{4}^{+}$ & $\mathrm{mg} \mathrm{NH}_{4}^{+\cdot} \mathrm{dm}^{-3}$ & 0.06 & 0.07 & 0.20 & 0.63 & 0.30 & 0.13 \\
\hline 11 & $\mathrm{TN}$ & $\mathrm{mg} \mathrm{N} \cdot \mathrm{dm}^{-3}$ & 1.49 & 1.58 & 1.43 & 2.39 & 2.18 & 1.67 \\
\hline 12 & SRP & $\mathrm{mg}-\mathrm{PO}_{4}{ }^{3-} \mathrm{dm}^{-3}$ & 0.10 & 0.11 & 0.10 & 0.16 & 0.17 & 0.16 \\
\hline 13 & $\mathrm{TP}$ & $\mathrm{mg} \mathrm{P}-\mathrm{PO}_{4}^{3--} \mathrm{dm}^{-3}$ & 0.38 & 0.40 & 0.36 & 0.60 & 0.67 & 0.17 \\
\hline 14 & $\mathrm{Ca}^{2+}$ & $\mathrm{mg} \mathrm{Ca} \cdot \mathrm{dm}^{-3}$ & 49 & 52 & 47 & 79 & 91 & 111 \\
\hline 15 & $\mathrm{Mg}^{2+}$ & $\mathrm{mg} \mathrm{Mg} \cdot \mathrm{dm}^{-3}$ & $<5$ & $<5$ & $<5$ & 6 & 7 & 8 \\
\hline 16 & $\mathrm{Cl}^{-}$ & $\mathrm{mg} \mathrm{Cl} \cdot \mathrm{dm}^{-3}$ & 17 & 18 & 16 & 27 & 23 & 28 \\
\hline 17 & $\mathrm{SO}_{4}^{2-}$ & $\mathrm{mg} \mathrm{SO}_{4} \cdot \mathrm{dm}^{-3}$ & 24 & 25 & 23 & 38 & 34 & 26 \\
\hline 18 & $\mathrm{HCO}_{3}^{-}$ & $\mathrm{mg} \mathrm{HCO}_{3} \cdot \mathrm{dm}^{-3}$ & 150 & 175 & 125 & 175 & 250 & 275 \\
\hline 19 & $\mathrm{Fe}_{\text {tot }}$ & $\mathrm{mg} \mathrm{Fe} \cdot \mathrm{dm}^{-3}$ & 0.43 & 0.45 & 0.41 & 0.69 & 0.60 & 0.40 \\
\hline 20 & $\mathrm{Mn}_{\text {tot }}$ & $\mathrm{mg} \mathrm{Mn} \cdot \mathrm{dm}^{-3}$ & 0.08 & 0.09 & 0.08 & 0.13 & 0.06 & 0.02 \\
\hline
\end{tabular}

Demonstrated dendrograms showing further gradual variation (Fig. 2) of water quality along the runoff path from $\mathrm{S}_{1}$ to $\mathrm{S}_{6}$. wherein can be seen clearly that between stations $\mathrm{S}_{4}-\mathrm{S}_{6}$ was always a distinct change of Osówka water quality. Tab. 1-4 shows that there has been a significant deterioration in the quality of the examined water irrespective of the date of measurement. Fig. 3 presented dendrogram showing the variation of the quality of the examined waters in terms from $T_{1}$ to $T_{4}$. It revealed that the examined waters in winter $\left(T_{1}\right)$ and summer $\left(T_{3}\right)$. when water temperatures were relatively stable - this variation was similar. whereas in terms $\mathrm{T}_{2}$ and $\mathrm{T}_{4}$ (spring and autumn) when significant changes occur in water temperatures in our climate zone - variability of examined indices was also - though different - similar. All collected data were used to calculate the next dendrogram on Fig. 4. which shows the variability of selected water chemistry indices. The dendrogram shows that there were certain groups of water quality indices. which changed in a similar way. These indices are very clearly grouped in several clusters which correspond to gradually changes in organic matter. oxygen conditions. $\mathrm{pH}$ and mineralization of the tested waters. and even show a correlation between changes in the concentrations of total 
iron and sulphates (VI). which might indicate. that the Osówka waters were contaminated with pollutants containing $\mathrm{FeSO}_{4}$. In turn. the linear regression equation showing effect of station number and date of the sampling on the variability of the examined indicators clearly show that the variability of Osówka water chemistry changing very substantially over time and along the path of the water runoff. because the in equations are virtually all stations from $\mathrm{S}_{1}$ to $\mathrm{S}_{6}$ and all sampling terms $\mathrm{T}_{1}-\mathrm{T}_{4}$. Thus. at each station. water quality is clearly different from the stations nearer or more distant from the station.

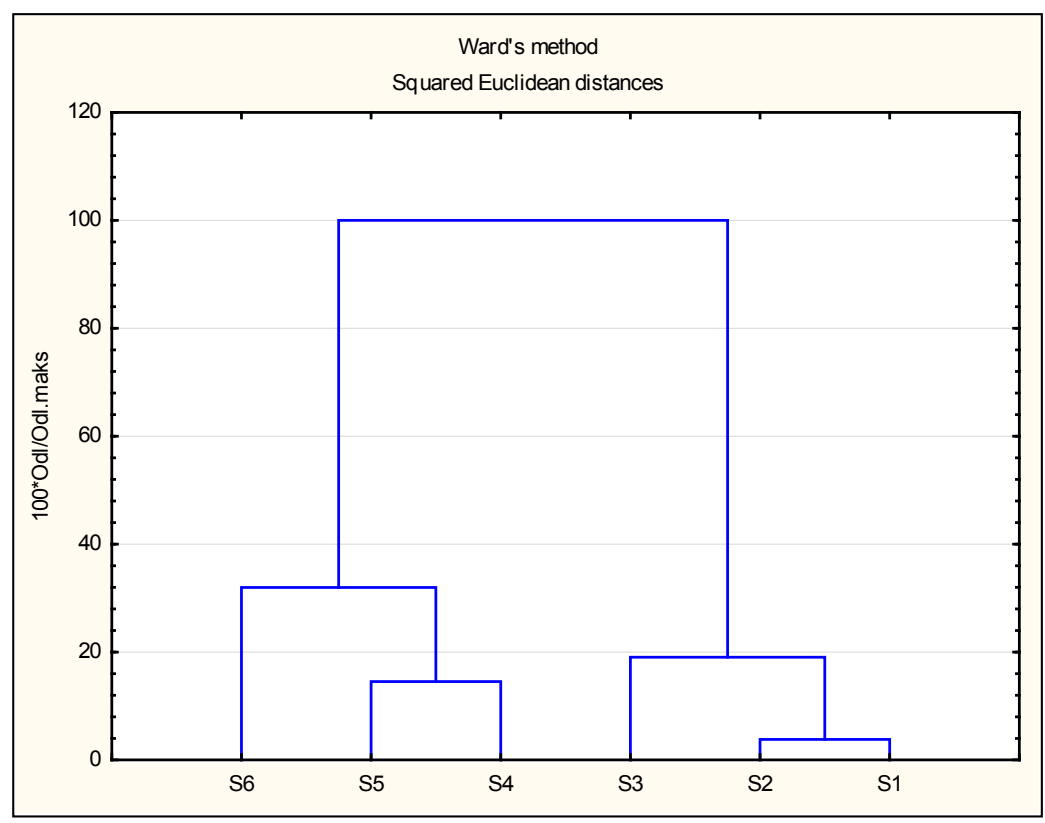

Figure 2. Dendrogram showing clustering of sampling stations in April and October 


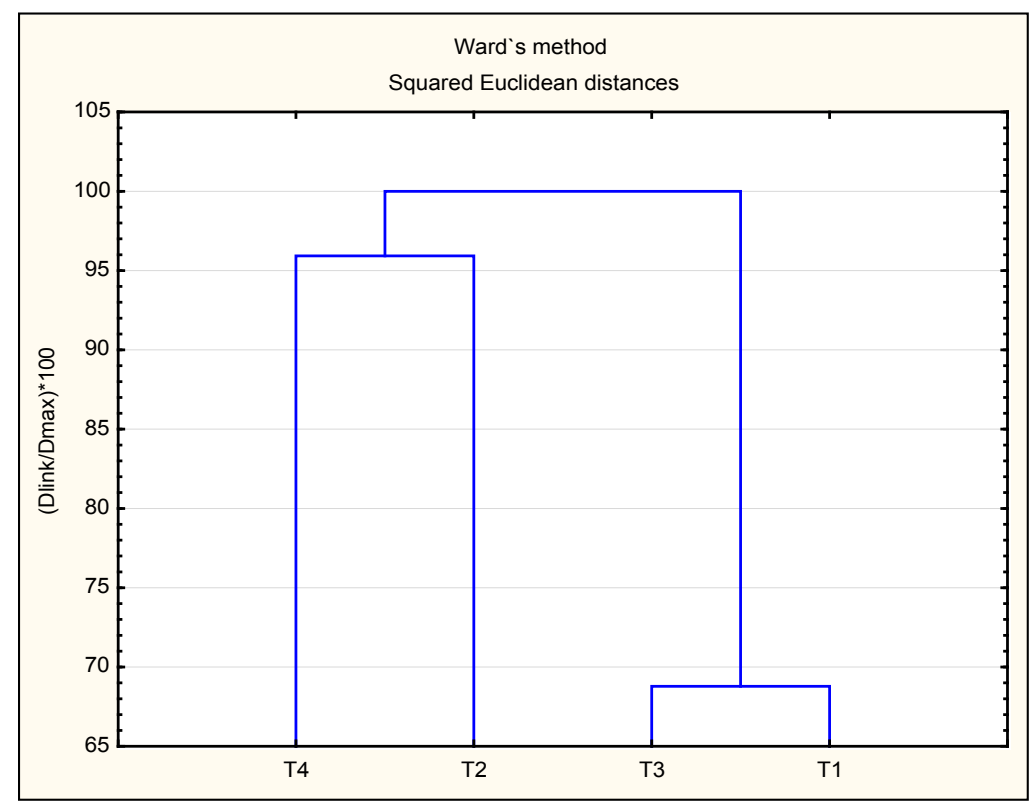

Figure 3. Dendrogram showing clustering of terms of sampling

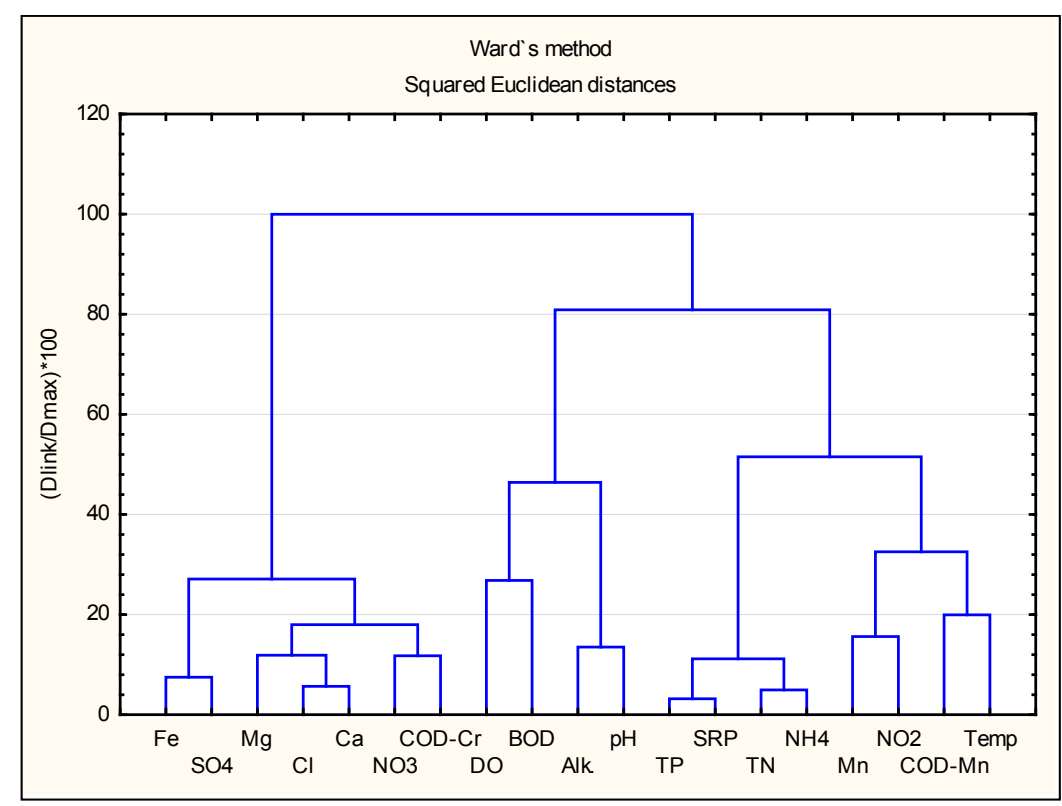

Figure 4. Relationship between selected water quality indices of Osówka stream in 2014 
Table 5. Linear regression equations showing the relationship between selected water quality indices $\left(\mathrm{y}_{\mathrm{i}}\right)$ from their mean values $\left(\bar{y}_{i}\right)$. sampling station location $\left(\mathrm{S}_{\mathrm{j}}\right)$ and term of sampling $\left(\mathrm{T}_{\mathrm{k}}\right)$

\begin{tabular}{|c|c|c|c|c|c|c|}
\hline No. & $\begin{array}{c}\text { Water } \\
\text { quality } \\
\text { indices }\left(y_{\mathrm{i}}\right)\end{array}$ & $y_{i}=\overline{y_{i}}+\sum_{j=1}^{6} \alpha_{j} S_{j}+\sum_{k=1}^{4} \beta_{k} T_{k}+S E E$ & \multicolumn{2}{|c|}{$\begin{array}{c}\text { Significance level } \\
\text { of coefficients in calculated } \\
\text { equation }\end{array}$} & SEE & $\mathrm{R}^{2}$ \\
\hline 1 & 2 & 3 & \multicolumn{2}{|c|}{4} & 5 & 6 \\
\hline 1 & Temperature & $\begin{array}{l}y=8.43+(11.94 \pm 0.96) \mathrm{T}_{1}+ \\
(-9.31 \pm 0.69) \mathrm{T}_{2}\end{array}$ & $\begin{array}{l}\alpha_{0}=0.0000 \\
\beta_{\mathrm{T} 1}=0.0000\end{array}$ & $\beta_{\mathrm{T} 2}=0.0000$ & 0.69 & 0.95 \\
\hline 2 & $\mathrm{pH}$ & $\begin{array}{l}y=7.97+(0.44 \pm 0.17) \mathrm{S}_{2}+ \\
(-0.37 \pm 0.17) \mathrm{S}_{4}+(0.31+0.17) \mathrm{S}_{6}+ \\
(0.36 \pm 0.17) \mathrm{T}_{3}\end{array}$ & $\begin{array}{l}\alpha_{0}=0.0000 \\
\alpha_{\mathrm{S} 4}=0.0184 \\
\alpha_{\mathrm{S} 6}=0.0436\end{array}$ & $\begin{array}{l}\beta_{\mathrm{T} 1}=0.0487 \\
\beta_{\mathrm{T} 3}=0.0201\end{array}$ & 0.17 & 0.64 \\
\hline 3 & COD-Mn & $\begin{array}{l}y=9.38+(0.69 \pm 0.29) \mathrm{S}_{4}+ \\
(1.14 \pm 0.29) \mathrm{S}_{6}+(-1.73 \pm 0.29) \mathrm{T}_{1}+ \\
(-0.67 \pm 0.29) \mathrm{T}_{3}\end{array}$ & $\begin{array}{l}\alpha_{0}=0.0000 \\
\alpha_{S 4}=0.0000 \\
\alpha_{S 6}=0.0001\end{array}$ & $\begin{array}{l}\beta_{\mathrm{T} 1}=0.0287 \\
\beta_{\mathrm{T} 3}=0.0147\end{array}$ & 0.29 & 0.82 \\
\hline 4 & $\mathrm{COD}-\mathrm{Cr}$ & $\begin{array}{l}y=106.00+(144.71 \pm 22.70) \mathrm{S}_{4}+ \\
(113.46 \pm 22.70) \mathrm{S}_{6}\end{array}$ & $\begin{array}{l}\alpha_{0}=0.0001 \\
\alpha_{S 4}=0.0000\end{array}$ & $\alpha_{\mathrm{S} 6}=0.0000$ & 22.70 & 0.74 \\
\hline 5 & BOD & $\begin{array}{l}y=2.89+(4.73 \pm 0.55) \mathrm{S}_{3}+ \\
(2.63 \pm 0.55) \mathrm{S}_{6}+(1.21 \pm 0.49) \mathrm{T}_{1}+ \\
(1.07 \pm 0.49) \mathrm{T}_{2}\end{array}$ & $\begin{array}{l}\alpha_{0}=0.0000 \\
\alpha_{\mathrm{S} 3}=0.0000 \\
\alpha_{\mathrm{S} 6}=0.0001\end{array}$ & $\begin{array}{l}\beta_{\mathrm{T} 1}=0.0024 \\
\beta_{\mathrm{T} 2}=0.0423\end{array}$ & 0.99 & 0.82 \\
\hline 6 & DO & $\begin{array}{l}y=6.97+(1.86 \pm 0.85) \mathrm{S}_{1}+ \\
(-8.23 \pm 0.85) \mathrm{S}_{4}+(-7.73 \pm 0.85) \mathrm{S}_{5}+ \\
(-4.28 \pm 0.85) \mathrm{S}_{6}+(2.50 \pm 0.69) \mathrm{T}_{2}\end{array}$ & $\begin{array}{l}\alpha_{0}=0.0002 \\
\alpha_{\mathrm{S} 1}=0.0433 \\
\alpha_{\mathrm{S} 4}=0.0000\end{array}$ & $\begin{array}{l}\alpha_{\mathrm{S} 5}=0.0000 \\
\alpha_{\mathrm{S} 6}=0.0000 \\
\beta_{\mathrm{T} 2}=0.0001\end{array}$ & 1.39 & 0.92 \\
\hline 7 & WS & $\begin{array}{l}y=56.00+(-65.82 \pm 7.23) \mathrm{S}_{4}+ \\
(-61.82 \pm 7.23) \mathrm{S}_{5}+(-31.78 \pm 7.23) \mathrm{S}_{6} \\
+(19.06 \pm 5.90) \mathrm{T}_{2}\end{array}$ & $\begin{array}{l}\alpha_{0}=0.0004 \\
\alpha_{S 4}=0.0000 \\
\alpha_{S 5}=0.0000\end{array}$ & $\begin{array}{l}\alpha_{\mathrm{S} 6}=0.0003 \\
\beta_{\mathrm{T} 2}=0.0004\end{array}$ & 11.81 & 0.92 \\
\hline 8 & $\mathrm{NO}_{3}^{-}$ & $\begin{array}{l}y=0.59+(0.50 \pm 0.11) \mathrm{S}_{4}+ \\
(0.44 \pm 0.11) \mathrm{S}_{5}+(0.39 \pm 0.11) \mathrm{S}_{6}\end{array}$ & $\begin{array}{l}\alpha_{0}=0.0000 \\
\alpha_{S 4}=0.0002\end{array}$ & $\begin{array}{l}\alpha_{\mathrm{SS}}=0.0009 \\
\alpha_{\mathrm{S} 6}=0.0039\end{array}$ & 0.19 & 0.67 \\
\hline 9 & $\mathrm{NO}_{2}^{-}$ & $\begin{array}{l}y=0.130+(0.131 \pm 0.022) \mathrm{S}_{4}+ \\
(-0.094 \pm 0.022) \mathrm{S}_{6}\end{array}$ & $\begin{array}{l}\alpha_{0}=0.0000 \\
\alpha_{S 4}=0.0000\end{array}$ & $\alpha_{\mathrm{S} 6}=0.0006$ & 0.03 & 0.82 \\
\hline 10 & $\mathrm{NH}_{4}^{+}$ & $\begin{array}{l}y=0.48+(0.29 \pm 0.07) \mathrm{S}_{4}+ \\
(0.44 \pm 0.07) \mathrm{S}_{5}+(0.39 \pm 0.07) \mathrm{S}_{6}+ \\
(-0.38 \pm 0.06) \mathrm{T}_{1}+(-0.46 \pm 0.06) \mathrm{T}_{4}\end{array}$ & $\begin{array}{l}\alpha_{0}=0.0074 \\
\alpha_{\mathrm{S} 4}=0.0000 \\
\alpha_{\mathrm{S} 5}=0.0000\end{array}$ & $\begin{array}{l}\alpha_{\mathrm{S} 6}=0.0001 \\
\beta_{\mathrm{T} 1}=0.0003 \\
\beta_{\mathrm{T} 4}=0.0114\end{array}$ & 0.12 & 0.87 \\
\hline 11 & TN & $\begin{array}{l}y=2.05+(1.06 \pm 0.13) \mathrm{S}_{4}+ \\
(0.91 \pm 0.13) \mathrm{S}_{5}+(0.62 \pm 0.13) \mathrm{S}_{6}+ \\
(-1.53 \pm 0.13) \mathrm{T}_{1}+(-0.90 \pm 0.13) \mathrm{T}_{4}\end{array}$ & $\begin{array}{l}\alpha_{0}=0.0466 \\
\alpha_{\mathrm{S} 4}=0.0000 \\
\alpha_{\mathrm{S} 5}=0.0000\end{array}$ & $\begin{array}{l}\alpha_{\mathrm{S} 6}=0.0000 \\
\beta_{\mathrm{T} 1}=0.0000 \\
\beta_{\mathrm{T} 4}=0.0001\end{array}$ & 0.22 & 0.95 \\
\hline 12 & SRP & $\begin{array}{l}y=0.23+(0.31 \pm 0.03) \mathrm{S}_{4}+ \\
(0.16 \pm 0.03) \mathrm{S}_{5}+(0.11 \pm 0.03) \mathrm{S}_{6}+ \\
(0.11 \pm 0.03) \mathrm{T}_{2}+(0.07 \pm 0.03) \mathrm{T}_{3}\end{array}$ & $\begin{array}{l}\alpha_{0}=0.0000 \\
\alpha_{S 4}=0.0000 \\
\alpha_{S 5}=0.0001\end{array}$ & $\begin{array}{l}\alpha_{\mathrm{S} 6}=0.0039 \\
\beta_{\mathrm{T} 2}=0.0041 \\
\beta_{\mathrm{T} 3}=0.0259\end{array}$ & 0.06 & 0.89 \\
\hline 13 & $\mathrm{TP}$ & $\begin{array}{l}y=0.77+(0.41 \pm 0.08) \mathrm{S}_{4}+ \\
(0.62 \pm 0.08) \mathrm{S}_{5}+(0.12 \pm 0.08) \mathrm{S}_{6}+ \\
(0.45 \pm 0.08) \mathrm{T}_{2}+(1.15 \pm 0.08) \mathrm{T}_{3}\end{array}$ & $\begin{array}{l}\alpha_{0}=0.0000 \\
\alpha_{S 4}=0.0000 \\
\alpha_{S 5}=0.0000\end{array}$ & $\begin{array}{l}\alpha_{\mathrm{S} 6}=0.0000 \\
\beta_{\mathrm{T} 2}=0.0001 \\
\beta_{\mathrm{T} 3}=0.0141\end{array}$ & 0.14 & 0.95 \\
\hline
\end{tabular}




\begin{tabular}{|c|c|c|c|c|c|c|}
\hline 1 & 2 & 3 & \multicolumn{2}{|c|}{4} & 5 & 6 \\
\hline 14 & $\mathrm{Ca}^{2+}$ & $\begin{array}{l}y=76.00+(22.47 \pm 4.17) \mathrm{S}_{4}+ \\
(40.97 \pm 4.17) \mathrm{S}_{5}+(63.72 \pm 4.17) \mathrm{S}_{6}+ \\
(-10.30 \pm 3.41) \mathrm{T}_{3}+(-10.80 \pm 3.41) \mathrm{T}_{4}\end{array}$ & $\begin{array}{l}\alpha_{0}=0.0000 \\
\alpha_{\mathrm{S} 4}=0.0005 \\
\alpha_{\mathrm{S} S}=0.0000\end{array}$ & $\begin{array}{l}\alpha_{\mathrm{S} 6}=0.0000 \\
\beta_{\mathrm{T} 3}=0.0005 \\
\beta_{\mathrm{T} 4}=0.0000\end{array}$ & 6.81 & 0.95 \\
\hline 15 & $\mathrm{Mg}^{2+}$ & $\begin{array}{l}y=17.00+(6.81 \pm 3.23) \mathrm{S}_{4}+ \\
(13.81 \pm 3.23) \mathrm{S}_{5}+(11.56 \pm 3.23) \mathrm{S}_{6}+ \\
(48.78 \pm 2.74) \mathrm{T}_{4}\end{array}$ & $\begin{array}{l}\alpha_{0}=0.0000 \\
\alpha_{S 4}=0.0493 \\
\alpha_{S 5}=0.0000\end{array}$ & $\begin{array}{l}\alpha_{\mathrm{S} 6}=0.0004 \\
\beta_{\mathrm{T} 4}=0.0021\end{array}$ & 5.59 & 0.95 \\
\hline 16 & $\mathrm{Cl}^{-}$ & $\begin{array}{l}y=24.00+(-1.76 \pm 0.77) \mathrm{S}_{3}+ \\
(10.19 \pm 0.77) \mathrm{S}_{4}+(6.44 \pm 0.77) \mathrm{S}_{5}+ \\
(10.44 \pm 0.77) \mathrm{S}_{6}+(6.27 \pm 0.73) \mathrm{T}_{2}+ \\
(3.16 \pm 0.73) \mathrm{T}_{3}\end{array}$ & $\begin{array}{l}\alpha_{0}=0.0000 \\
\alpha_{\mathrm{S} 3}=0.0367 \\
\alpha_{\mathrm{S} 4}=0.0000 \\
\alpha_{\mathrm{S} 5}=0.0000\end{array}$ & $\begin{array}{l}\alpha_{\mathrm{S} 6}=0.0000 \\
\beta_{\mathrm{T} 2}=0.0005 \\
\beta_{\mathrm{T} 3}=0.0000\end{array}$ & 1.26 & 0.97 \\
\hline 17 & $\mathrm{SO}_{4}^{2-}$ & $\begin{array}{l}y=37.00+(30.29 \pm 7.37) \mathrm{S}_{4}+ \\
(26.29 \pm 7.37) \mathrm{S}_{5}+(18.29 \pm 7.37) \mathrm{S}_{6}+ \\
(-12.62 \pm 6.02) \mathrm{T}_{4}\end{array}$ & $\begin{array}{l}\alpha_{0}=0.0000 \\
\alpha_{\mathrm{S} 4}=0.0005 \\
\alpha_{\mathrm{S} 5}=0.0020\end{array}$ & $\begin{array}{l}\alpha_{\mathrm{S} 6}=0.0226 \\
\beta_{\mathrm{T} 4}=0.0497\end{array}$ & 12.76 & 0.61 \\
\hline 18 & Alkalinity & $\begin{array}{l}y=205.00+(-45.00 \pm 11.91) \mathrm{S}_{3}+ \\
(-16.25 \pm 11.91) \mathrm{S}_{4}+(35.00 \pm 11.91) \mathrm{S}_{5} \\
+(67.50 \pm 11.91) \mathrm{S}_{6}+(-20.27 \pm 9.17) \\
\mathrm{T}_{3}\end{array}$ & $\begin{array}{l}\alpha_{0}=0.0000 \\
\alpha_{\mathrm{S} 3}=0.0319 \\
\alpha_{\mathrm{S} 4}=0.0001\end{array}$ & $\begin{array}{l}\alpha_{\mathrm{S} 5}=0.0008 \\
\alpha_{\mathrm{S} 6}=0.0005 \\
\beta_{\mathrm{T} 3}=0.0402\end{array}$ & 16.46 & 0.83 \\
\hline 19 & $\mathrm{Fe}_{\text {tot }}$ & $\begin{array}{l}y=0.47+(0.50 \pm 0.13) \mathrm{S}_{4}+ \\
(0.43 \pm 0.13) \mathrm{S}_{5}+(-0.11 \pm 0.11) \mathrm{T}_{3}\end{array}$ & $\begin{array}{l}\alpha_{0}=0.0000 \\
\alpha_{S 4}=0.0011\end{array}$ & $\begin{array}{l}\alpha_{\mathrm{S} 5}=0.0036 \\
\beta_{\mathrm{T} 3}=0.0065\end{array}$ & 0.24 & 0.53 \\
\hline 20 & $\mathrm{Mn}_{\text {tot }}$ & $\begin{array}{l}y=0.06+(0.04 \pm 0.01) \mathrm{S}_{4}+ \\
(-0.04 \pm 0.01) \mathrm{S}_{6}+(-0.05 \pm 0.01) \mathrm{T}_{1}\end{array}$ & $\begin{array}{l}\alpha_{0}=0.0000 \\
\alpha_{\mathrm{S} 4}=0.0146\end{array}$ & $\begin{array}{l}\alpha_{\mathrm{S}}=0.0052 \\
\beta_{\mathrm{T} 1}=0.0000\end{array}$ & 0.02 & 0.77 \\
\hline
\end{tabular}

Notation: Values of $y_{i}$ are received after substitution for the appropriate $S_{j}$ and $T_{k}-$ the value 1

To determine which water quality indices should be examined in order to record the variability of water quality of Osówka stream. and also to verify that the parameters $S_{j}$ and $T_{k}$ also prejudge that water should be tested in all selected measuring stations - factor analysis were conducted. The analysis results are shown in Tab. 6 . for all the stations and terms of sampling. It was assumed that the important parameters for which the coefficients in Tab. 6 are $\geq 0.70$. It was established - in such way - that the parameters characterizing the variability of the examined waters are: among the general parameters - temperature. $\mathrm{pH}$. DO. COD-Cr and COD-Mn. WS. $\mathrm{BOD}_{5}$. from indices characterizing the content of biogenic substances - all test indices. i.e.. $\mathrm{NO}_{3}$. $\mathrm{NO}_{2}^{-} \cdot \mathrm{NH}_{4}^{+}$. TN. SPR. TP. from the indices characterizing the mineralization - concentrations: $\mathrm{Ca}^{2+} \cdot \mathrm{Mg}^{2+} \cdot \mathrm{Cl}^{-}$and $\mathrm{HCO}_{3}{ }^{-}$. At the same time FA showed importance of station number $\left(\mathrm{S}_{\mathrm{j}}\right)$ and sampling date $\left(\mathrm{T}_{\mathrm{k}}\right)$ - which confirms our earlier findings made with Tab. 5. Of course the value of assumed classification criterion as eg. recommended by Liu et al. 2003 can be increased to value 0.75 and the important parameters would be: temperature. $\mathrm{pH}$. $\mathrm{BOD}_{5}$. $\mathrm{NO}_{3}^{-} \cdot \mathrm{NO}_{2}^{-} \cdot \mathrm{NH}_{4}^{+}$. TN . SRP. TP. $\mathrm{Ca}^{2+} \cdot \mathrm{Mg}^{2+}$. $\mathrm{S}_{\mathrm{j}}$ and $\mathrm{T}_{\mathrm{k}}$. While such changes would prevent the conduction of studies on the biohydrogeochemical processes taking place in examined waters eg. equilibria of processes of organic matter mineralization (Yu et al. 2015). or balance processes between the forms of nitrogen (Lusk. Toor 2016). as well as studies on the concentrations of ion macronutrients in water (Halstead 2014). The case determines the value of classification criterion as above may also be a matter of judgment. 
Table 6. Factor analysis after varimax rotation for Osówka Stream in four seasons of 2014 year

\begin{tabular}{|c|c|c|c|c|c|}
\hline & FV1 & FV2 & FV3 & FV4 & FV5 \\
\hline Temperature & -0.18 & 0.15 & 0.06 & 0.86 & 0.28 \\
\hline $\mathrm{pH}$ & -0.07 & 0.25 & 0.83 & 0.10 & 0.20 \\
\hline COD-Mn & 0.25 & 0.21 & 0.03 & 0.72 & -0.28 \\
\hline COD-Cr & 0.70 & 0.22 & -0.25 & 0.11 & -0.11 \\
\hline $\mathrm{BOD}_{5}$ & 0.04 & -0.17 & 0.09 & -0.13 & -0.90 \\
\hline DO & -0.71 & -0.35 & 0.28 & -0.17 & -0.12 \\
\hline WS & 0.66 & 0.55 & -0.17 & -0.07 & 0.08 \\
\hline $\mathrm{NO}_{3}^{-}$ & -0.05 & 0.21 & -0.81 & 0.19 & 0.41 \\
\hline $\mathrm{NO}_{2}^{-}$ & 0.21 & 0.91 & 0.06 & -0.06 & -0.11 \\
\hline $\mathrm{NH}_{4}^{+}$ & 0.23 & 0.87 & -0.06 & 0.33 & 0.04 \\
\hline $\mathrm{TN}$ & 0.16 & 0.84 & 0.02 & 0.18 & 0.14 \\
\hline SRP & 0.06 & 0.91 & 0.03 & 0.12 & 0.21 \\
\hline $\mathrm{TP}$ & 0.91 & 0.14 & 0.30 & 0.03 & -0.08 \\
\hline $\mathrm{Ca}^{2+}$ & 0.82 & -0.22 & 0.06 & -0.30 & 0.15 \\
\hline $\mathrm{Mg}^{2+}$ & 0.87 & 0.20 & -0.10 & -0.19 & -0.07 \\
\hline $\mathrm{Cl}^{-}$ & 0.71 & 0.33 & -0.39 & -0.26 & 0.19 \\
\hline $\mathrm{SO}_{4}^{2-}$ & 0.64 & 0.10 & 0.69 & -0.01 & 0.14 \\
\hline $\mathrm{HCO}_{3}^{-}$ & 0.43 & 0.09 & -0.73 & -0.11 & 0.29 \\
\hline $\mathrm{Fe}_{\text {tot }}$ & -0.48 & 0.30 & -0.30 & 0.29 & 0.22 \\
\hline Station & 0.87 & 0.22 & 0.10 & 0.20 & -0.28 \\
\hline Term of sampling & -0.16 & 0.06 & -0.03 & 0.94 & 0.13 \\
\hline Expl.Var & 6.02 & 4.18 & 2.89 & 2.74 & 1.62 \\
\hline Prp.Totl & 0.29 & 0.20 & 0.14 & 0.13 & 0.08 \\
\hline Eigenvalue & 7.18 & 4.27 & 2.98 & 1.75 & 1.25 \\
\hline$\%$ Total & 34.21 & 20.32 & 14.20 & 8.33 & 5.97 \\
\hline $\begin{array}{l}\text { Cumulative } \\
\text { Eigenvalue }\end{array}$ & 7.18 & 11.45 & 14.43 & 16.18 & 17.44 \\
\hline Cumulative \% & 34.21 & 54.54 & 68.73 & 77.07 & 83.04 \\
\hline
\end{tabular}

\section{Conclusion}

1. The study showed that the Osówka stream waters are characterized by high variability along the road runoff. from the station near to Osówka source to stream inflow to the Rusalka lake. The biggest changes of quality occurred between the station 3 and 4 - ie. between the Goplana lake ponds Syrenie Stawy. 
2. When examining the variability of water quality Osówka - if such monitoring would be carried out - should be mean. among the general indices - temperature. $\mathrm{pH}$. DO. COD-Cr. COD-Mn. WS. BOD ${ }_{5}$ and as regards the biogenic substances : $\mathrm{NO}_{3}^{-} \cdot \mathrm{NO}_{2}{ }^{-} \cdot \mathrm{NH}_{4}^{+}$. TN. SPR. TP; and in terms of mineral substances: $\mathrm{Ca}^{2+} \cdot \mathrm{Mg}^{2+} \cdot \mathrm{Cl}^{-}$and $\mathrm{HCO}_{3}{ }^{-}$. All the above data seem to be necessary for a thorough determination of the water quality variability and to collect data to indicate what biogeohydrochemical processes occurred in examined waters in the Osówka ecosystem. Among the 20 designated water quality indices of Osówka stream - should be examine - by our findings - 16 indices when the water sampling would be made. as it was done at 6 measuring stations located along the runoff path in terms for 4 seasons. In our opinion. sampling of water in more sampling stations and more than 4 times a year would be advisable.

\section{References}

APHA. 2012. Standard methods for examination of water and wastewater. $18^{\mathrm{h}}$ ed. Washington. p. 1800.

Chang H. 2008. Spatial analysis of water quality trends in the Han River basin. South Korea. Wat. Res., 42 (13): 3285-3304. DOI: 10.1016/j.watres.2008.04.006.

Halstead J.A., Kliman S., Berheid C.W., Chaucer A., Cock-Esteb A. 2014. Urban stream syndrome in a small lightly developed watershed: a statistical analysis of water chemistry parameters, land use patterns, and natural sources. Environ. Monit. Assess., 186 (6): 3391-3414. DOI: 10.1007/s10661-0143625-9.

Hamid A., Bhat S.A., Bhat S.U., Jehangir A. 2016. Environmetric techniques in water quality assessment and monitoring: a case study. Environ Earth Sci., 75: 321. DOI:10.1007/s12665-015-5139-3.

Haque M.Z., Rahim S.A., Abdullah M.P., Embi A.F., Elfithri R., Lihan T., Wan Mohd Khalik W.M.A., Khan M.F., Mokhtar M. 2016. Multivariate chemometric approach on the surface water quality in langat upstream tributaries, peninsular Malaysia. J. Environ. Sci. Technol., 9 (3): 277-284. DOI: 10.3923/ jest.2016.277.284.

Huang Z., Han L., Zeng L., Xiao W., Tian Y. 2016. Effects of land use patterns on stream water quality: a case study of a small-scale watershed in the Three Gorges Reservoir Area. China. Environ. Sci. Pollut. Res., 23 (4): 3943-3955. DOI: 10.1007/s11356-015-5874-8.

Ledford S.H., Lautz L.K. 2015. Floodplain connection buffers seasonal changes in urban stream water quality. Hydrol. Process., 29 (6): 1002-1016. DOI: 10.1002/hyp.10210.

Liu C.W., Lin K.H., Kuo Y.M. 2003. Application of factor analysis in the assessment of groundwater quality in a blackfoot disease area in Taiwan. Sci. Total Environ., 313 (1): 77-89. DOI: 10.1016/S00489697(02)00683-6.

Liu J.S., Guo L.C., Luo X.L., Chen F.R., Zeng E.Y. 2014. Impact of anthropogenic activities on urban stream water quality: a case study in Guangzhou. China. Environ. Sci. Pollut. Res., 21 (23): 13412 13419. DOI: 10.1007/s11356-014-3237-5.

Lusk M.G., Toor G.S. 2016. Dissolved organic nitrogen in urban streams: Biodegradability and molecular composition studies. Water Research., 96: 225-235. DOI: 10.1016/j.watres.2016.03.060.

Miller T., Poleszczuk G., Wawrzyniak W., Drozdowska A., Tokarz M., Tymanowski Ł. 2014b. Ciek Osówka w aglomeracji miejskiej Szczecina - zmiany jakości wód wzdłuż drogi spływu od źródła do jeziora Rusałka. In: Bezpieczeństwo Publiczne - BP'14. T. Zaborowski. (ed.). Wyd. Instytut Badań i Ekspertyz Naukowych. Poznań-Gorzów Wielkopolski. pp. 236-252. DOI: 10.13140/2.1.4980.8963.

Miller T., Tokarz M., Poleszczuk G. 2014a. Stream Osówka in Szczecin - chemometric analysis of water quality indices in winter season. Acta Biologica, 21: 91-104. 
Muangthong S., Shrestha S. 2015. Assessment of surface water quality using multivariate statistical techniques: case study of the Nampong River and Songkhram River. Thailand. Environ. Monit. Assess., 187 (9): 1-12. DOI: 10.1007/s10661-015-4774-1.

Nemerow N.L. 1985. Stream. lake. estuary and ocean pollution. Van Nostrand Reinhold. New York.

Polish Standards. Water quality.

Pratt B., Chang H. 2012. Effects of land cover, topography, and built structure on seasonal water quality at multiple spatial scales. J. Hazard. Mat., 209: 48-58. DOI: 10.1016/jjhazmat.2011.12.068.

Raczyńska M., Grzeszczyk-Kowalska A., Raczyński M. 2012a. Zastosowanie metody River Habitat Survey do waloryzacji hydromorfologicznej cieku Osówka (Pomorze Zachodnie). Inż. Ekol., 30: 266-276.

Raczyńska M., Machula S., Raczyński M. 2012b. Materia organiczna jako ważny element stopnia zanieczyszczenia małych cieków na przykładzie potoku Osówka. Inż. Ekol., 31: 91-100.

Report o stanie Miasta Szczecina. 2004: 105-114. Available: http://www.szczecin.pl/raport04/html/8_0. htm.

Stan Środowiska Miasta Szczecina (stan na 31 grudnia 2002 roku). Opracowany w ramach „Programu ochrony dla miasta Szczecina". 2004. Available: http://www.ikzm-oder.de/download.php?fileid=2150.

Stanisz A. 2007. Przystęny kurs statystyki z zastosowaniem Statisica PL na przyktadach z medycyny. Tom 2: Modele liniowe i nieliniowe. Wyd. StatSoft. Kraków. p. 868.

Sun N., Yearsley J., Baptiste M., Cao Q., Lettenmaier D.P., Nijssen B. 2016. A spatially distributed model for assessment of the effects of changing land use and climate on urban stream quality. Hydrol. Process. DOI: 10.1002/hyp.10964.

Uchwała Rady Miasta Szczecina Nr L/708/94 z dnia 16 maja 1994 r. w sprawie uznania niektórych terenów za użytki ekologiczne i zespoły przyrodniczo-krajobrazowe. 1994. Available: http://bip.um.szczecin. pl/UMSzczecinBIP/files/16F3148550C44C2B8BAF768CBEB21709/708-94.pdf.

Wilkins P.M., Cao Y., Heske E.J., Levengood J.M. 2015. Influence of a forest preserve on aquatic macroinvertebrates, habitat quality, and water quality in an urban stream. Urban Ecosyst., 18 (3): 989-1006. DOI: $10.1007 / \mathrm{s} 11252-015-0464-6$.

Yu H., Song Y., Gao H., Liu L., Yao L., Peng J. 2015. Applying fluorescence spectroscopy and multivariable analysis to characterize structural composition of dissolved organic matter and its correlation with water quality in an urban river. Environ. Earth Sci.,73 (9): 5163-5171. DOI: 10.1007/s12665-0154269 -y.

Cite as: Miller T., Poleszczuk G. 2016. Urban stream Osówka in Szczecin - how efficiently as possible should the variability of the stream water quality be studied along the road of runoff in time? Acta Biologica, 23: 55-68. DOI: 10.18276/ab.2016.23-05. 